\title{
Smoothening Mechanism of Thiourea on Silver Electrodeposition. Real Time Imaging of the Growth Front Evolution
}

\author{
O. Azzaroni, P. L. Schilardi, R. C. Salvarezza, and A. J. Arvia* \\ Instituto de Investigaciones Fisicoquímica Teóricas y Aplicadas (INIFTA), Facultad de \\ Ciencias Exactas, Universidad Nacional de La Plata, CONICET, Sucursal 4, Casilla de \\ Correo 16, (1900) La Plata, Argentina
}

Received May 26, 1998. In Final Form: October 21, 1998

\begin{abstract}
Ag electrodeposits were grown on a polycrystalline Ag cathode at either 0.01 or $1 \mathrm{~mA} \mathrm{~cm}^{-2}$ from aqueous $5 \times 10^{-3} \mathrm{M} \mathrm{Ag}_{2} \mathrm{SO}_{4}+10^{-2} \mathrm{M} \mathrm{H}_{2} \mathrm{SO}_{4}+0.5 \mathrm{M} \mathrm{Na}_{2} \mathrm{SO}_{4}$, in the absence and presence of different amounts of thiourea (TU). The cathode/solution interface evolution in a quasi-bidimensional electrochemical cell was followed with a video microscope imaging system. From TU-free solution a nodular morphology at $0.01 \mathrm{~mA} \mathrm{~cm}^{-2}$ and a branched morphology at $1 \mathrm{~mA} \mathrm{~cm}^{-2}$ were observed. At $0.01 \mathrm{~mA} \mathrm{~cm}^{-2}$ the presence of TU drove the interface motion to a stable roughness regime by suppressing the nucleation and growth of instabilities. Accordingly, the characteristics of the growth front were consistent with the predictions of the Edwards-Wilkinson equation for the interface motion. At $1 \mathrm{~mA} \mathrm{~cm}^{-2}$, those electrodeposits grown in the presence of TU showed a decrease in both the nucleation and growth rates of instabilities, although they were not fully suppressed.
\end{abstract}

\section{Introduction}

Metal electrodeposition can be used to prepare films with growth patterns ranging from self-similar fractal to rough compact patterns by setting appropriate growth rate conditions, such as the applied electric potential, the composition of the plating bath, hydrodynamic conditions in the system, and temperature. ${ }^{1}$ In general, at high current densities, branched deposits with unstable growth fronts can be produced assisted by the presence of Laplacian fields. ${ }^{2-4}$

In electrochemical systems, instabilities at the growth front can be suppressed by adding certain organic molecules (additives) to the plating solution. The smoothness and brightness of $\mathrm{Ni}, \mathrm{Zn}, \mathrm{Sn}$, and $\mathrm{Cu}$ electrodeposits can be substantially improved when a certain amount of additive is present in the plating solution. ${ }^{5,6}$ In principle, additive molecules can either be adsorbed or electroadsorbed on the surface of the growing deposit, and to some extent, can even be incorporated into it. Intensive practical uses of additives in metal-plating baths have been made for a long time without having a convincing physicochemical support for their performance. This means that a comprehensive theory describing the kinetics and mechanisms of metal electrodeposition from additive-containing plating baths is still lacking. ${ }^{7}$

In the past decade, great efforts have been made to describe the evolution of growing interfaces by means of

* To whom correspondence should be addressed. Fax: 54-21254642. E-mail: ajarvia@isis.unlp.edu.ar.

(1) Matshushita, M. In The Fractal Approach to the Heterogeneous Chemistry, Avnir, D., Ed.; John Wiley: New York, 1989; p 161.

(2) Stanley, E.; Barabasi, A. Fractal Concepts in Surface Growth: Cambridge University Press: New York, 1995, and references therein

(3) Meakin. P. In The Fractal Approach to the Heterogeneous Chemistry, Avnir, D., Ed.; John Wiley: New York, 1989; p 131.

(4) Meakin, P. Phys. Rep. 1993, 235, 191, and references therein.

(5) Modern Electroplating, Lowenheim, F. A., Ed.; Wiley-Interscience: Princenton, NJ, 1974.

(6) Despic, A. R. In Comprehensive Treatise of Electrochemistry, Conway, B. E., Bockris, J. O.'M., Yaeger, E., Khan, S. U. M., White, R. E., Eds.; Plenum Press: New York, 1983; Vol. 7, p 451.

(7) Barkey, D. P.; Muller, R. H.; Tobias, C. W. J. Electrochem. Soc. 1989, $136,2207$. continuous models. In general, these models start from a plane substrate and describe the interface evolution by a partial differential equation ${ }^{2}$ that involves at least two counterbalancing contributions, one resulting from the stochastic noise of finite-sized depositing particles and the other from surface relaxation phenomena. The state of the art on this matter shows that the agreement of models and experimental data is still far from satisfactory. ${ }^{8}$ Conversely, data from the growth mode of $\mathrm{Cu}$ electrodeposits, produced from additive-containing plating baths under a predominantly charge-transfer rate control, ${ }^{9}$ showed that $\partial h / \partial t$, the growth rate of the interface width, $h(x, t)$, resulting from the analysis of ex-situ atomic force microscopy (AFM) image profiles, could be represented by the following linear differential equation: ${ }^{10,11}$

$$
\partial h(x, t) / \partial t=\sigma \nabla^{2} h(x, t)-K \nabla^{4} h(x, t)+\eta(x, t)
$$

In eq $1, \sigma$ is the surface tension coefficient and $K$ is a constant related to the surface diffusion coefficient of depositing particles. The first linear term in the right hand side (rhs) member corresponds to a surface relaxation contribution operating through adsorption-desorption-like processes, ${ }^{11}$ and the second linear term refers to surface relaxation by surface diffusion. ${ }^{10}$ The term $\eta(x, t)$ stands for the white Gaussian noise related to the aggregation of finite-sized particles. The competition between linear terms generates a horizontal characteristic length defined by $L_{\mathrm{c}}=(K / \sigma)^{1 / 2}$, so that for a scaling length $L<L_{\mathrm{c}}$, the interface evolution is dominated by the second term of eq 1 , leading to the Villain-Wolf rate equation, ${ }^{10}$ whereas for $L>L_{\mathrm{c}}$, the first term in eq 1 determines the scaling

(8) Krim, J.; Palasantzas, G. Int. J. Modern Phys. B 1995, 9, 599 (9) Vázquez, L.; Salvarezza, R. C.; Arvia, A. J. Phys. Rev. Lett. 1997, 79, 709. Mendez, S.; Andreasen, G.; Schilardi, P.; Figueroa, M.; Vázquez, L.; Salvarezza, R. C.; Arvia, A. J. Langmuir 1998, 14, 2515. Schilardi, P.; Mendez, S.; Salvarezza, R. C.; Arvia, A. J. Langmuir 1998, 14, 4308

(10) Wolf, D.; Villain, J. Europhys. Lett. 1990, 13, 389. Villain, J. J. Phys. I 1992, 1.

(11) Edwards, S. F.; Wilkinson, D. R. Proc. R. Soc. London A 1982 381,17 . 
behavior of the system leading to the Edwards-Wilkinson (EW) rate equation. ${ }^{11}$ In fact, for a complex system such as that involved in $\mathrm{Cu}$ electrodeposition at a current density mainly associated with a charge-transfer overpotential, it was found that the evolution of the growth front could be described on the asymptotic limit by the EW equation. As this result is rather surprising, it is sound to attempt to verify the validity of the EW equation for other metal electrodeposition processes in which a different kinetic control is involved.

This work deals with real time growth front evolution for Ag electrodeposition from acid solution baths either with or without the addition of thiourea (TU) at 0.01 and $1 \mathrm{~mA} \mathrm{~cm}-2$, and at $298 \mathrm{~K}$, using a quasi-bidimensional (2D) cell. In TU-free solution the growth front becomes unstable, yielding either a nodular or a branched morphology at low and high current density, respectively. The nodular morphology is favored by a concentration field built up around the electrodeposit, whereas branching is favored under the combined effect of electric and concentration fields. For Ag electrodeposits formed at 0.01 $\mathrm{mA} \mathrm{cm}{ }^{-2}$, the presence of TU suppresses the growth of instabilities by hindering the capture of $\mathrm{Ag}^{+}$ions at protrusions and favoring Ag electrodeposition at valleys. This picture is consistent with the physics involved in the EW equation. Otherwise, for those electrodeposits grown at $1 \mathrm{~mA} \mathrm{~cm}{ }^{-2}$, the presence of TU diminishes both the nucleation and growth rates of instabilities without suppressing them completely because of the high rate of $\mathrm{Ag}^{+}$ion discharging that prevents adsorption equilibrium for TU.

\section{Experimental Section}

Ag electrodeposits were grown at $298 \mathrm{~K}$ under constant apparent current density, namely, $j=0.01 \mathrm{~mA} \mathrm{~cm}^{-2}$ and $j=1$ $\mathrm{mA} \mathrm{cm}{ }^{-2}$, from aqueous $5 \times 10^{-3} \mathrm{M} \mathrm{Ag}_{2} \mathrm{SO}_{4}+10^{-2} \mathrm{M} \mathrm{H}_{2} \mathrm{SO}_{4}+$ $0.5 \mathrm{M} \mathrm{Na}_{2} \mathrm{SO}_{4}+\mathbf{x}(0 \leq \mathbf{x} \leq 0.1) \mathrm{mM}$ TU solutions.

Plating solutions were prepared from analytical reagent quality chemicals and MilliQ-Millipore* water. They were saturated with purified $\mathrm{N}_{2}$ gas by flowing it through the solution for $2 \mathrm{~h}$ before each electrochemical run.

The quasi-2D electrochemical cells consisted of a parallel electrode arrangement of a polycrystalline Ag cathode (geometric area $\approx 1 \mathrm{~cm}^{2}$ ) and a Ag anode (geometric area $\approx 4.2 \mathrm{~cm}^{2}$ ). These electrodes were made from a $0.024 \mathrm{~cm}$ thick Ag strip $(99.99 \%$ purity). The anode-to-cathode distance in these cells was set to $d_{\mathrm{ac}}=1.3 \mathrm{~cm}$.

Cathodic polarization curves were recorded at the potential scan rate $s=10^{-2} \mathrm{~V} \mathrm{~s}^{-1}$ to approach to quasi-steady-state electrodeposition conditions and produce a small change in the surface area of Ag electrodeposits. ${ }^{12}$ Data from these curves were plotted as either $\Delta E_{\mathrm{c}}$ vs $j$ or as $\Delta E_{\mathrm{c}}$ vs $\log j$, where $\Delta E_{\mathrm{c}}$ is the cathodic potential between anode and cathode.

The evolution of the topography of each Ag electrodeposit was followed at $298 \mathrm{~K}$ using a sequential video-microscope imaging system with a $750 \times 570$ pixel resolution. Images were taken in the range $0.8 \leq L \leq 1.30 \mathrm{~mm}$. The images shown in this paper are representative of the overall electrodeposit morphology. The electrodeposit morphologies exhibit no edge effects attributable to a nonuniform current distribution.

\section{Results and Interpretation.}

3.1. Electrochemical Data. Polarization curves for Ag electrodeposition on the Ag substrate, in the range 0 $\leq \Delta E_{\mathrm{c}} \leq 1.2 \mathrm{~V}$, depend on TU concentration. The cathodic polarization curve in the absence of TU $(\mathbf{x}=0)$ (Figure 1a) exhibits three well-defined regions (I, II, and III). Region I covers the range $0 \leq \Delta E_{\mathrm{c}} \leq 0.1 \mathrm{~V}$ in which the

(12) Encyclopedia of Electrochemistry of the Elements; Bard, A. J., Ed.; M. Dekker: New York, 1973; Vol. 8, p 2.
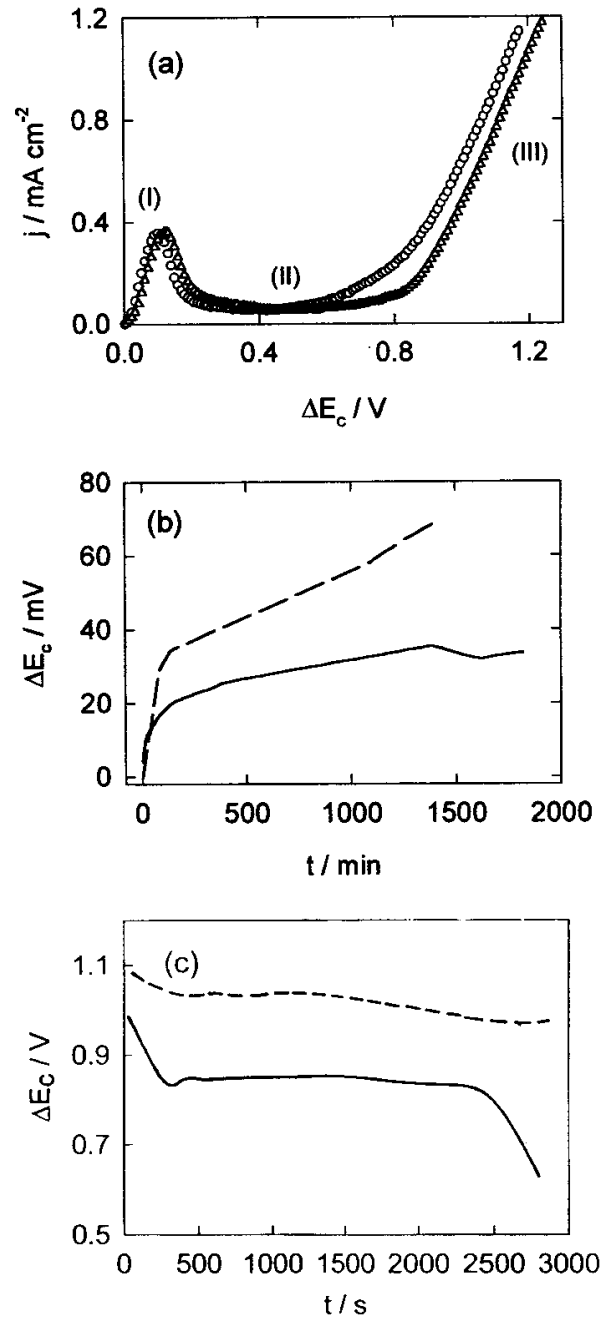

Figure 1. (a) Cathodic polarization curves at $2 \times 10^{-3} \mathrm{~V} \mathrm{~s}^{-1}$ for Ag electrodeposition on $\mathrm{Ag}$ from $\left(5 \times 10^{-3} \mathrm{M} \mathrm{Ag}_{2} \mathrm{SO}_{4}+10^{-2}\right.$ $\mathrm{M} \mathrm{H}_{2} \mathrm{SO}_{4}+0.5 \mathrm{M} \mathrm{Na}_{2} \mathrm{SO}_{4}+\mathbf{x ~ m M ~ T U}$, at $298 \mathrm{~K}$, using a quasi$2 D$ cell. $(O) \mathbf{x}=0 ;(\triangle) \mathbf{x}=0.1$. Regions I, II, and III are indicated in the figure. (b) $\Delta E_{\mathrm{c}}$ vs $t$ plots for $j=0.01 \mathrm{~mA} \mathrm{~cm}^{-2}$ resulting from $5 \times 10^{-3} \mathrm{M} \mathrm{Ag}_{2} \mathrm{SO}_{4}+10^{-2} \mathrm{M} \mathrm{H}_{2} \mathrm{SO}_{4}+0.5 \mathrm{M} \mathrm{Na}_{2} \mathrm{SO}_{4}+$ $\mathbf{x} \mathrm{mM}$ TU, at $298 \mathrm{~K}$, using a quasi-2D cell. (Full trace) $\mathbf{x}=0$. (dashed trace) $\mathbf{x}=0.1$. (c) $\Delta E_{\mathrm{c}}$ vs $t$ plots for $j=1 \mathrm{~mA} \mathrm{~cm} \mathrm{~cm}^{-2}$ resulting from $5 \times 10^{-3} \mathrm{M} \mathrm{Ag}_{2} \mathrm{SO}_{4}+10^{-2} \mathrm{M} \mathrm{H}_{2} \mathrm{SO}_{4}+0.5 \mathrm{M}$ $\mathrm{Na}_{2} \mathrm{SO}_{4}+\mathbf{x} \mathrm{mM}$ TU, at $298 \mathrm{~K}$, using a quasi-2D cell. (Full trace) $\mathbf{x}=0$; (dashed trace) $\mathbf{x}=0.1$.

Ag electrodeposition reaction is kinetically controlled by the nucleation and growth of isolated crystals under diffusion control, as was concluded from the typical loop that has been reported from polarization curves recorded at $0.02 \mathrm{~V} \mathrm{~s}^{-1}$ using a conventional 3D cell. ${ }^{13}$ In this case, $\Delta E_{\mathrm{c}} \cong \eta_{\mathrm{c}}$, where $\eta_{\mathrm{c}}$ represents the crystallization overpotential. ${ }^{13}$ On the other hand, for $0.1 \mathrm{~V} \leq \Delta E_{\mathrm{c}} \leq 0.5 \mathrm{~V}$, the cathodic current first reaches a maximum that depends on $s$, and then attains a limiting current (region II) as expected for an electrochemical reaction under a steady mass-transport rate control. The overlapping of diffusion layers, built up around growing crystals in region II, results in a diffusion-controlled kinetics for $\mathrm{Ag}$ electrodeposition, and in this case, $\Delta E_{\mathrm{d}} \cong \eta_{\mathrm{d}}$, where $\eta_{\mathrm{d}}$ is a concentration overpotential. Finally, for $\Delta E_{\mathrm{c}}>0.5 \mathrm{~V}$, the current increases markedly with $\Delta E_{\mathrm{c}}$ (region III) ${ }^{13}$ because of the triggering of large Ag trees at Ag crystal borders, the size of these trees being larger than the local diffusion layer thickness there, as discussed further on.

(13) Hernández Creus, H.; Carro, P.; González, S.; Salvarezza, R. C. Arvia, A. J. Electrochim. Acta 1992, 37, 2215. 
(a)
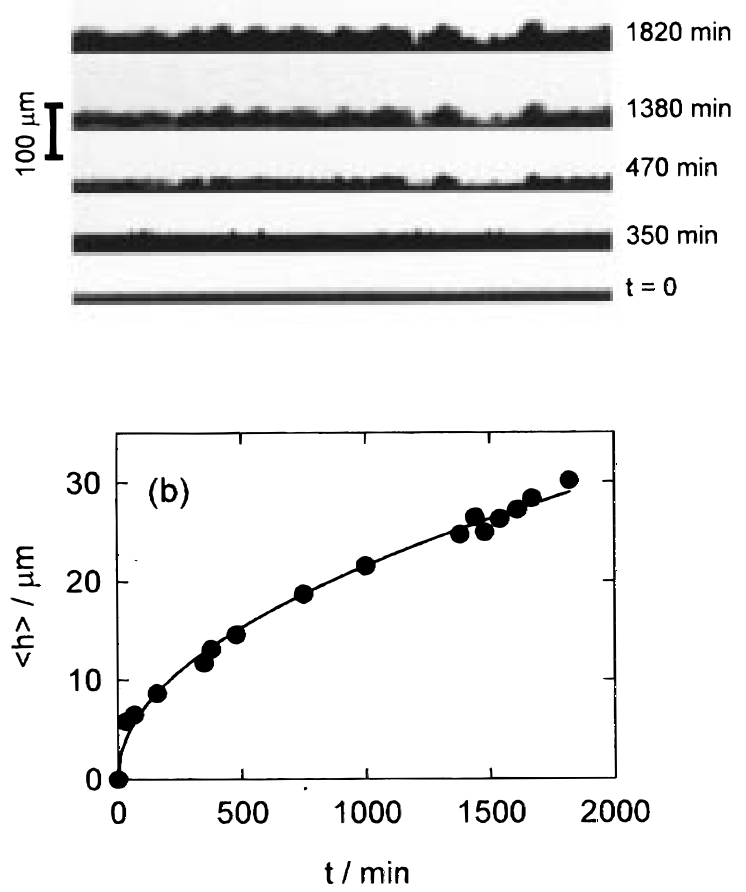

Figure 2. (a) In situ lateral micrographs showing the interface evolution from $t=0$ to $t=1820 \mathrm{~min}$ of a Ag electrodeposit formed at $j=0.01 \mathrm{~mA} \mathrm{~cm}{ }^{-2}$ from $5 \times 10^{-3} \mathrm{M} \mathrm{Ag}_{2} \mathrm{SO}_{4}+10^{-2}$ $\mathrm{M} \mathrm{H}_{2} \mathrm{SO}_{4}+0.5 \mathrm{M} \mathrm{Na}_{2} \mathrm{SO}_{4}$, using quasi-2D cell at $298 \mathrm{~K}$. (b) $\langle h\rangle$ vs $t$ plot resulting from the analysis of profiles shown in (a).

For $j=0.01 \mathrm{~mA} \mathrm{~cm} \mathrm{~cm}^{-2}$, the value of $\Delta E_{\mathrm{c}}$ increases progressively from 0.004 to $0.033 \mathrm{~V}$ (Figure 1b), whereas for $j=1 \mathrm{~mA} \mathrm{~cm}^{-2}, \Delta E_{\mathrm{c}}$ first decreases from $1.0(t=0)$ to $0.82 \mathrm{~V}(t=5 \mathrm{~min})$ (Figure 1c), remains constant for $t=$ $40 \mathrm{~min}$, and finally, decreases to $0.6 \mathrm{~V}(t=50 \mathrm{~min})$.

Cathodic polarization curves for Ag electrodeposition from TU-containing solutions recorded in the same potential range $\left(0 \leq \Delta E_{\mathrm{c}} \leq 1.5 \mathrm{~V}\right)$ and $\mathrm{Ag}^{+}$-ion concentration (Figure 1a) show differences in regions I and III when compared to those curves resulting from a TU-free solution. In these regions, as $\mathbf{x}$ is increased, the value of $\Delta E_{\mathrm{c}}$ that is required for attaining a steady value of $j$ also increases (Figure 1a). The fact that region II remains unchanged in the presence of TU indicates that the additive does not affect the mass-transport resistance from the solution side (i.e., the current density in region II approaches a common limiting current density value $\left.\left(\mathrm{j}_{1}\right)\right)$, irrespective of $\mathbf{x}$.

In a TU-containing solution at $j=0.01 \mathrm{~mA} \mathrm{~cm}^{-2}$ (Figure 1b) and $j=1 \mathrm{~mA} \mathrm{~cm}^{-2}$ (Figure 1c) time-dependent values of $\Delta E_{\mathrm{c}}$ lying in regions I and III, respectively, are observed. These values of $\Delta E_{\mathrm{c}}$ are always greater than those resulting from the TU-free solution.

Therefore, from the analysis of polarization data it can be concluded that the major influence of $\mathrm{TU}$ on $\mathrm{Ag}$ electrodeposition appears as an additional resistance operating at the surface level.

3.2. Lateral Imaging of Growing Electrodeposits. 3.2.1. Electrodeposits Grown at $\boldsymbol{j}=0.01 \mathrm{~mA} \mathbf{c m}^{-2}$. The sequential imaging of Ag electrodeposits produced from the TU-free solution at $j=0.01 \mathrm{~mA} \mathrm{~cm}^{-2}$ (region I) shows the progressive nucleation and growth of nodular crystals which finally overlap (Figure 2a). The average height of the deposit $(\langle h\rangle)$, defined as $\langle h\rangle=\Sigma h_{i} / n$, with $n$ being the number of points of height $h_{i}$, increases with time according to $\langle h\rangle \propto t^{0.5}$ (Figure $2 \mathrm{~b}$ ). This type of relationship is expected for a deposit grown under mass-
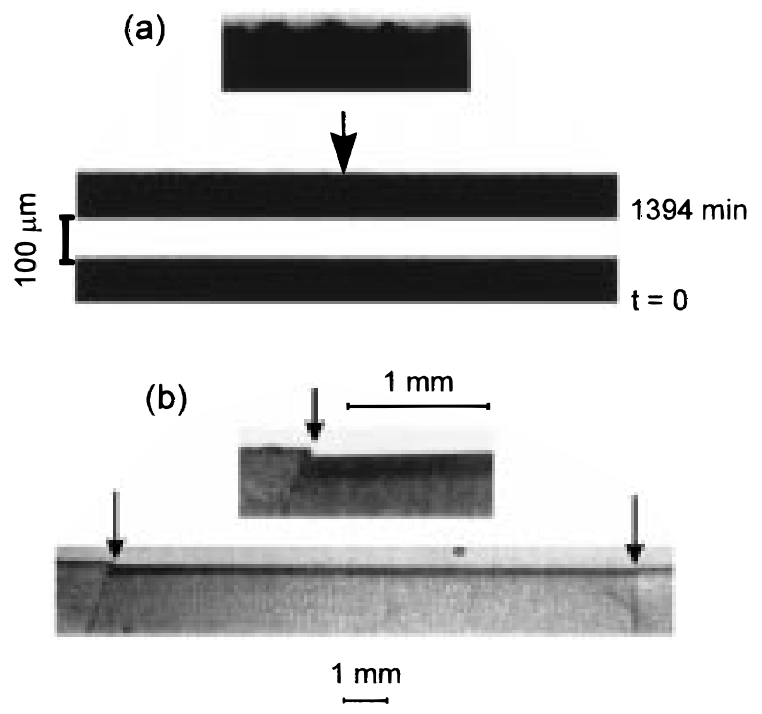

Figure 3. (a) In situ lateral micrographs showing the interface evolution from $t=0$ to $t=1394$ min of a Ag electrodeposit built up at $j=0.01 \mathrm{~mA} \mathrm{~cm}{ }^{-2}$ from $5 \times 10^{-3} \mathrm{M} \mathrm{Ag}_{2} \mathrm{SO}_{4}+10^{-2} \mathrm{M} \mathrm{H}_{2} \mathrm{SO}_{4}$ $+0.5 \mathrm{M} \mathrm{Na}_{2} \mathrm{SO}_{4}+0.1 \mathrm{mM}$ TU, using quasi-2D cell at $298 \mathrm{~K}$. (b) Lateral image of the electrode (length between arrows) after Ag electrodeposition for 1000 min. A smooth Ag deposit is grown without evidences of edge effects (see the arrow in the magnified detail of the picture).

transport control from the solution side ${ }^{14}$ and constant apparent area (i.e., $\langle h\rangle\langle\langle\delta\rangle$, where $\langle\delta\rangle$ stands for the average thickness of the diffusion layer. The velocity of the growth front $(V)$ is dominated by the enlargement of large crystals capturing most of the electrodepositing $\mathrm{Ag}^{+}$ ions leading to nodule formation, and the development of an unstable growth front, at least in the time range covered by this work. Therefore, the thickness of the growth front $(w)$, which becomes proportional to the size of the largest crystals, also increases with $t$ as $W \propto t^{0.5}$. Accordingly, the $V \propto t^{-0.5}$ relationship is also found. Then, these results confirm that Ag electrodeposition in region I is under masstransport control.

The evolution of the growth front changes drastically when Ag electrodeposits are produced from TU-containing solutions. In this case, as $\mathbf{x}$ is increased from 0 to 0.1 , the formation of large nodular crystals is completely suppressed, leading to a smooth electrode surface (Figure 3a,b). At higher magnifications, small fluctuations associated with local instabilities can also be observed. In contrast to Ag electrodeposits produced from a TU-free solution, these instabilities decay rapidly and finally disappear. Despite the fact that at our experimental-scale length the increase in $\langle h\rangle$ with time cannot be measured because the average thickness of the compact Ag electrodeposit, as estimated by Faraday law, is only $3.1 \times$ $10^{-4} \mathrm{~cm}$, it is evident that in our case the overall deposit appears smooth and free of edge effects that might have been produced by a nonuniform current distribution (Figure $3 b$ ). Then, it can be concluded that the stable interface growth results from the presence of TU in the solution.

3.2.2. Electrodeposits Grown at $\boldsymbol{j}=1 \mathrm{~mA} \mathrm{~cm}^{-2}$. The sequential imaging of Ag electrodeposits produced from the TU-free solution at $j=1 \mathrm{~mA} \mathrm{~cm}{ }^{-2}$ (region III) first shows the formation of irregular patterns that initially consist of a nearly compact dendrite-structured layer about $0.01 \mathrm{~cm}$ thick. This is followed by the progressive growth

(14) Deuch, J. M.; Meakin, P. J. Chem. Phys. 1984, 80, 2115. 
(a)
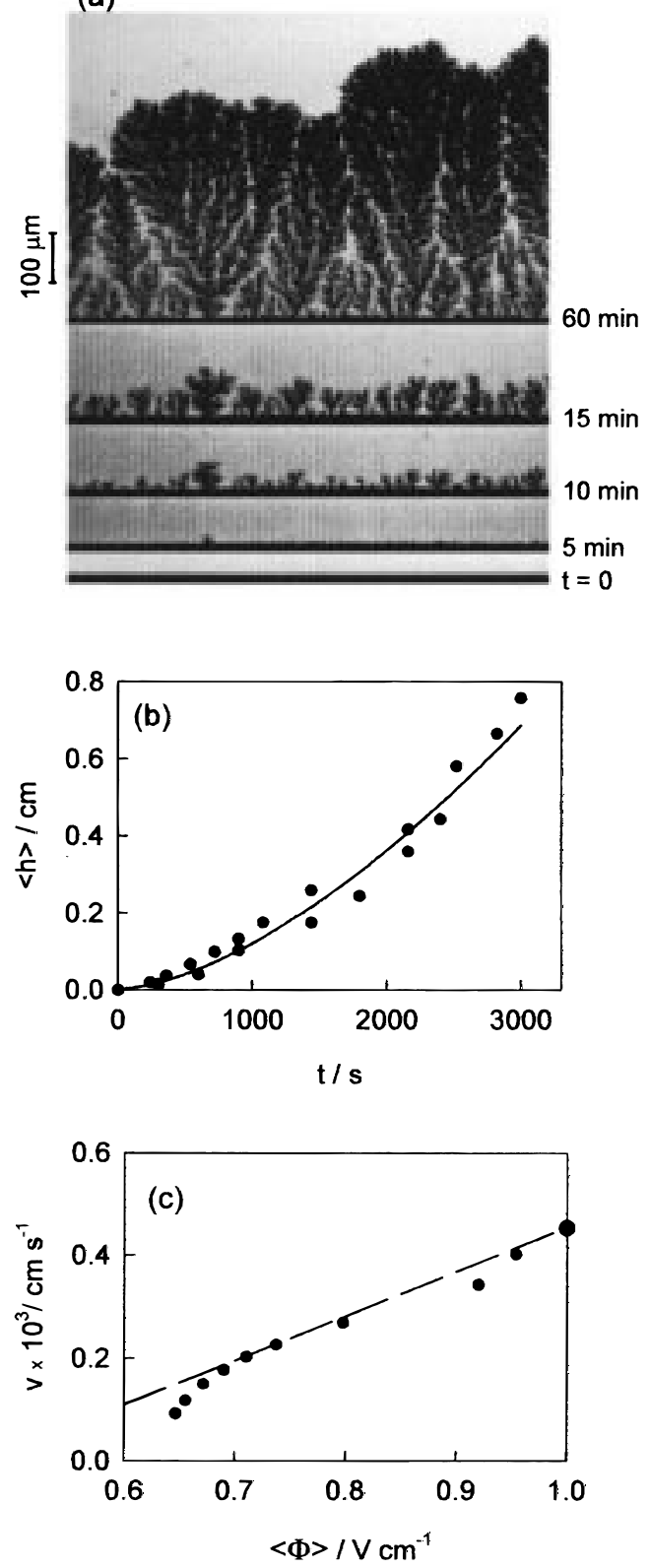

Figure 4. (a) In situ lateral micrographs showing the interface evolution from $t=0$ to $t=60 \mathrm{~min}$ for Ag electrodeposition at $j=1 \mathrm{~mA} \mathrm{~cm}{ }^{-2}$ in $5 \times 10^{-3} \mathrm{M} \mathrm{Ag}_{2} \mathrm{SO}_{4}+10^{-2} \mathrm{M} \mathrm{H}_{2} \mathrm{SO}_{4}+0.5 \mathrm{M}$ $\mathrm{Na}_{2} \mathrm{SO}_{4}$ using a quasi-2D cell at $298 \mathrm{~K}$. (b) $\langle h\rangle$ vs $t$ plot resulting from the analysis of profiles shown in (a). (c) $v$ vs $\langle\Phi\rangle$ plot. The slope of the straight line (arbitrarily located on the $y$-axis) indicates the mobility of sulfate anions.

of isolated large Ag trees at certain dendrite sites (Figure $4 a)$. At this stage of growth, from the evolution of the largest trees, it follows that $\langle h\rangle \propto t^{1.8}$ (Figure $4 \mathrm{~b}$ ) and $V \propto$ $t^{0.8}$. Similar results have been reported for $\mathrm{Cu}$ electrodeposition at high current densities. ${ }^{15}$ Then, the value of $W$ is dominated by the growth of large trees capturing most of the electrodepositing $\mathrm{Ag}^{+}$ions, whereas a large number of initially growing sites become practically frozen (Figure 4a,b). The result is the development of an unstable growth front. The development of unstable growth fronts has been related to the presence of electric and concentration fields assisting metal growth at protrusions that are normal to the deposit surface. ${ }^{2}$ Finally, at advanced stages of growth, the overlapping of large trees occurs

(15) Pastor, J. M.; Rubio, M. A. Phys. Rev. Lett. 1996, 76, 1848. and a change from an unstable to a stable growth front can be observed. ${ }^{16}$ In this case, a close inspection of growth fronts, such as that shown in Figure 4 a for $t=60 \mathrm{~min}$, reveals features similar to those generated by ballistic and Eden models. ${ }^{17}$

Let us first consider the contribution of the concentration field to the interface motion. The value of $\langle\delta\rangle$ can be estimated from the equation $\langle\delta\rangle=D / v$, taking the diffusion coefficient of $\mathrm{Ag}^{+}$ions in the solution $D=1 \times 10^{-5} \mathrm{~cm}^{2}$ $\mathrm{s}^{-1},{ }^{18}$ and $v=1.7 \times 10^{-4} \mathrm{~cm} \mathrm{~s}^{-1}$ (i.e., the value of $\mathrm{v}$ measured in the interval $10 \leq t \leq 20$ min when the system approaches a quasi-linear $h$ vs $t$ dependence (Figure $4 \mathrm{~b})$ ). Then, it results in $\delta=0.06 \mathrm{~cm}$, a figure that is much smaller than the size of large trees. Accordingly, only branches or trees smaller than $0.06 \mathrm{~cm}$ grow under the main influence of the concentration field.

On the other hand, large emerging trees $(\langle h\rangle>\langle\delta\rangle)$ (Figure 4a) exhibit a dense morphology with a fractal dimension $D_{f}=1.8$, a value predicted by Monte Carlo simulations of DLA patterns of a size larger than $\langle\delta\rangle{ }^{14}$ However, if this was exactly the case, then the motion of the growth front would follow a $\langle h\rangle \propto t$ relationship ${ }^{14,19}$ involving a constant $v$ rather than $h \propto t^{1.8}$ and $V \propto t^{0.8}$ relationships (Figure 4 b). This discrepancy would indicate that other relevant driving forces, such as the contribution of the electric field, participate in the electrodeposition process.

The influence of the electric field on the motion of the growth front can be determined through the dependence of $V$ on the electric field $(\Phi)$. At each electrodeposition time, the value of $v$ results from $v=\mathrm{d}\langle h\rangle / \mathrm{d} t$ (Figure $4 \mathrm{~b}$ ), while the average electric field $(\langle\Phi\rangle)$ can be estimated from $\langle\Phi\rangle=\Delta E_{\mathrm{c}}(t) /\left(d_{\mathrm{ac}}-\langle h\rangle\right)$ (Figure 1c). For $\langle\Phi\rangle<0.7 \mathrm{~V} \mathrm{~cm}^{-1}$, the $\langle v\rangle$ vs $\langle\Phi\rangle$ plot (Figure 4c) shows that v increases fast with $\langle\Phi\rangle$, whereas for $\langle\Phi\rangle>0.7 \mathrm{~V} \mathrm{~cm}^{-1}, V$ approaches a linear dependence on $\langle\Phi\rangle^{20}$ with a slope close to that resulting from the sulfate anion mobility in the solution. This is clear evidence that, for the solution composition used in this work, the electric field plays a relevant role in the motion of the growth front of large trees during Ag electrodeposition.

The interface evolution observed in a TU-free solution also appears for those Ag electrodeposits formed from TUcontaining solutions at $j=1 \mathrm{~mA} \mathrm{~cm}^{-2}$. However, in these cases, both the number of trees $(N)$ and their size decrease sharply as $\mathbf{x}$ is increased from 0 to 0.06 (Figure $5 \mathrm{a}$ ). This is evident by plotting $\rho_{\mathrm{N}}$ vs $t$, where $\rho_{\mathrm{N}}$ denotes the density of crystals (Figure $5 \mathrm{~b}$ ). This plot shows two regions, in the first one the slope $\mathrm{d} N / \mathrm{d} t$ (i.e., the rate of tree formation) diminishes as $\mathbf{x}$ is increased, and in the second one, after a certain time, a limiting value of $N$ is reached. This limiting value of $N$ becomes independent of $\mathbf{x}$, although the time required to reach it decreases as $\mathbf{x}$ is increased. Nevertheless, both the $\langle h\rangle \propto t^{1.8}$ (Figure 5c), and the $V$ vs $\langle\Phi\rangle$ plots behave similarly to those already described for Ag electrodeposits produced from TU-free solution (Figure $5 \mathrm{~d})$. It is evident that in contrast to Ag electrodeposition

(16) Castro M.; Cuerno, R.; Sánchez, A.; Dominguez-Adame, F. Phys. Rev. E. 1998, 57, R2491.

(17) Schilardi, P.; Azzaroni, O.; Salvarezza, R. C.; Arvia A. J. Phys. Rev. B., in press.

(18) Carro, P.; Ambrosolio, S.; Marchiano, S. L.; Salvarezza, R. C Hernández Creus, A.; Arvia, A. J. J. Electroanal. Chem. 1995, 396, 183.

(19) Ferer, M.; Smith, D. H. Phys. Rev. E. 1994, 49, 4114.

(20) Fleury, V.; Chazalviel J. N.; Rosso, N.; Sapoval, B. J. Electroanal. Chem. 1990, 290, 249. 
(a)
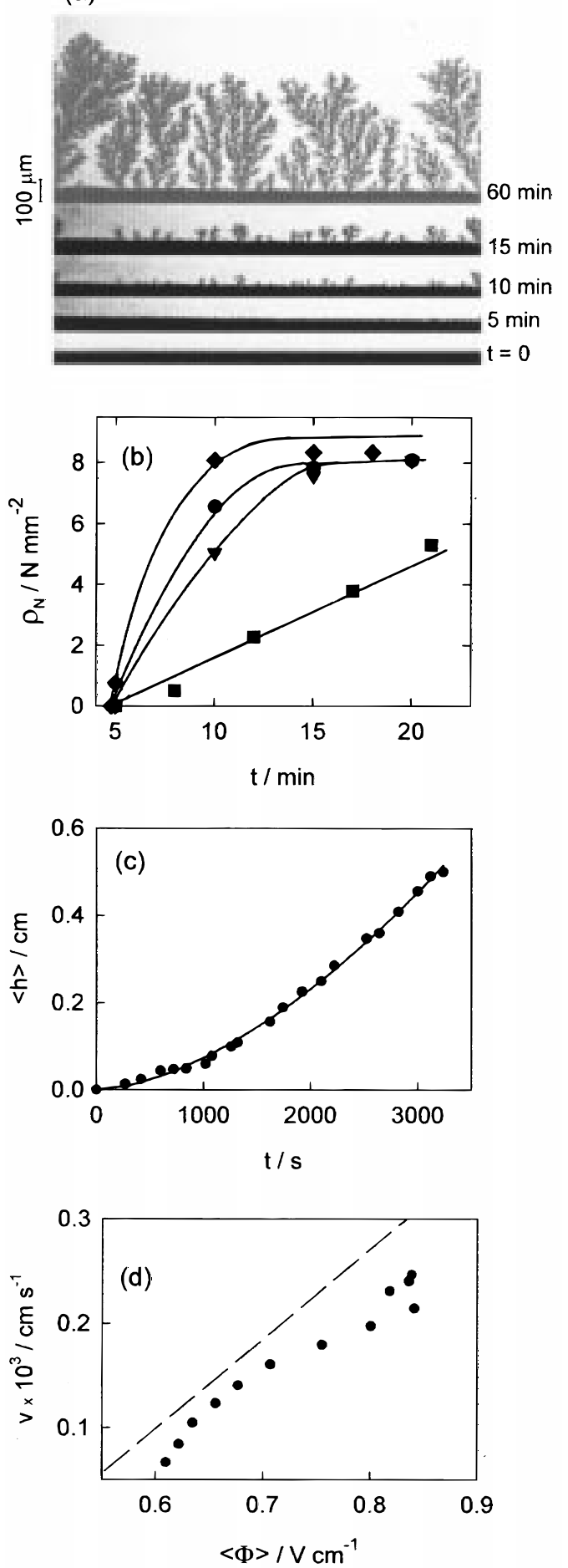

Figure 5. (a) In situ lateral micrographs showing the interface evolution from $t=0$ to $t=60 \mathrm{~min}$ for Ag electrodeposition at $j=1 \mathrm{~mA} \mathrm{~cm}^{-2}$ in $5 \times 10^{-3} \mathrm{M} \mathrm{Ag}_{2} \mathrm{SO}_{4}+10^{-2} \mathrm{M} \mathrm{H}_{2} \mathrm{SO}_{4}+0.5 \mathrm{M}$ $\mathrm{Na}_{2} \mathrm{SO}_{4}+0.06 \mathrm{mM}$ TU using a quasi-2D cell at $298 \mathrm{~K}$. (b) $\rho_{\mathrm{N}}$ vs $t$ plot, $\rho_{\mathrm{N}}$. $(\diamond) x=0 \mathrm{mM} ;(\bullet) x=0.02 ;(\nabla) x=0.04 ;(\mathbf{\square}) x=$ 0.06 . (c) $\langle h\rangle$ vs $t$ plot resulting from the analysis of profiles shown in (a). (d) $V$ vs $\langle\Phi\rangle$ plot. The slope of the straight line (arbitrarily located on the $y$-axis) indicates the mobility of sulfate anions

at $j=0.01 \mathrm{~mA} \mathrm{~cm}^{-2}$ and similar values of $\mathbf{x}$, at $j=1 \mathrm{~mA}$ $\mathrm{cm}^{-2}$ the presence of TU cannot completely suppress instabilities, although they are to some extent hindered.

\section{Discussion}

4.1. Ag Electrodeposition from Thiourea-Free Solution. The electrodeposition of Ag from TU-free solution containing an excess of a supporting electrolyte at $0.01 \mathrm{~mA} \mathrm{~cm}{ }^{-2}$ can be described by the following equations:

$$
\begin{gathered}
\mathrm{Ag}^{+}(\text {sol }) \rightarrow \mathrm{Ag}^{+} \text {(surf) } \\
\mathrm{e}^{-}+\mathrm{Ag}^{+}(\text {surf }) \rightarrow \mathrm{Ag}(\text { surf })
\end{gathered}
$$

Reaction 2 corresponds to the transport of $\mathrm{Ag}^{+}$ions from the bulk of the solution to the growing surface. The maximum steady mass-transport rate constant under free convection, at $298 \mathrm{~K}$, can be estimated as $5 \times 10^{-4} \mathrm{~cm} \mathrm{~s}^{-1}$. Reaction 3 represents the electrochemical discharge of $\mathrm{Ag}^{+}$ions at the electrode surface, increasing the electrodeposited Ag phase. The rate of this reaction under conditions of equilibrium, unit $\mathrm{Ag}^{+}$ion concentration, and $298 \mathrm{~K}$, on polycrystalline $\mathrm{Ag}$, is given by the exchange current density, $j_{0}=1 \mathrm{~A} \mathrm{~cm}^{-2},{ }^{12}$ a figure that decreases almost linearly with the $\mathrm{Ag}^{+}$ion concentration in the solution. The value of $j_{0}$ is equivalent to the electrodeposition of about 3000 monolayers of Ag per second (ML $\left.\mathrm{s}^{-1}\right)$.

For the $\mathrm{Ag}^{+}$ion concentrations used in this work, the transport of the $\mathrm{Ag}^{+}$ion in the solution becomes ratedetermining (reaction 2). At $0.01 \mathrm{~mA} \mathrm{~cm}^{-2}\left(\approx 0.03 \mathrm{ML} \mathrm{s}^{-1}\right)$ the growth process undergoes inside the diffusion layer and the morphology of the deposit is modeled by the concentration field. ${ }^{3}$ Under these conditions the growth of protrusions is enhanced and, in this case, the development of diffusion-limited aggregation (DLA)-like patterns would be expected. However, in the time range covered by this work no typical DLA-like morphology is observed probably because of the high surface mobility of $\mathrm{Ag}$ adatoms at the Ag/solution interface. ${ }^{21}$ In fact, Monte Carlo simulations of DLA models including surface diffusion relaxation shows a change from DLA to columnar, and finally to smooth patterns as the diffusion length (I) for depositing particles is increased. ${ }^{22}$ In fact, using $l=D_{\mathrm{s}} / F^{2}$, $D_{\mathrm{s}}$ being the surface diffusion coefficient for Ag adatoms, $F$, the flux, and taking $D_{\mathrm{s}}=1 \times 10^{-12} \mathrm{~cm}^{2} \mathrm{~s}^{-123}$ and $F=$ $1 \times 10^{-9} \mathrm{~cm} \mathrm{~s}^{-1}\left(0.03 \mathrm{ML} \mathrm{s}^{-1}\right)$, it results in $l=10^{-3} \mathrm{~cm}$. It should be noted that coarsening of $\mathrm{Au}$ particles from the $\mathrm{nm}$ to the $\mu \mathrm{m}$ range as well as rough Au surface smoothening produced via a surface adatom diffusion mechanism have been observed in acid solution at room temperature. These processes take place within the same time range involved in our $\mathrm{Ag}$ electrodeposition runs at low $j$ values. ${ }^{24}$ Therefore, it is reasonable to admit that in the long electrodeposition time range Ag surface mobility contributes to eliminating surface irregularities in the order of several microns.

Surface diffusion is a curvature-driven process which tends to enhance the attachment of particles at valleys. ${ }^{2}$ These local processes can be represented by

$$
\begin{gathered}
\operatorname{Ag}^{+}(\text {sol }) \rightarrow \operatorname{Ag}^{+}(- \text {surf }) \\
\mathrm{e}^{-}+\mathrm{Ag}^{+}(- \text {surf }) \rightarrow \operatorname{Ag}(- \text { surf }) \\
\operatorname{Ag}(- \text { surf }) \rightarrow \operatorname{Ag}(+ \text { surf })
\end{gathered}
$$

Reactions $2^{\prime}$ and $3^{\prime}$ stand for the enhanced electrodeposition at negatively curved surfaces (-surf) (protrusions)

(21) Martins, M. E.; Hernández Creus, A.; Salvarezza, R. C.; Arvia, A. J. J. Electroanal. Chem. 1994, 375,141.

(22) Salvarezza, R. C.; Alonso, C.; Vara, J. M.; Albano, E.; Martin, H. O.; Arvia, A. J. Phys. Rev. B, 1990, 41, 12502.

(23) Martins, M. E.; Hernandez-Creus, A.; Salvarezza, R. C.; Arvia, A. J.; J. Electroanal. Chem. 1994, 375, 141.

(24) Alonso, C.; Salvarezza, R. C.; Vara, J. M.; Arvia, A. J.; Vazquez, L.; Bartolome A.; Baro, A. M.; J. Electrochem. Soc. 1990, 137, 2161 
due to the presence of Laplacian fields, while reaction 4 represents the curvature-driven surface diffusion of $\mathrm{Ag}$ adatoms from negatively to positively curved surfaces (+surf) (valleys). In fact, in our system, for $j=0.01 \mathrm{~mA}$ $\mathrm{cm}^{-2}$, instabilities are only partially smoothed by reaction 4 , leading to an unstable nodular growth front. This indicates that the rate of both reactions 2 ' and $3^{\prime}$ exceeds that of reaction 4 , although the contribution of the latter to eliminate branching cannot be disregarded in the time range of our experiments.

On the other hand, for $j=1 \mathrm{~mA} \mathrm{~cm}^{-2}\left(\approx 3 \mathrm{ML} \mathrm{s}^{-1}\right)$, it results only in $l=10^{-5} \mathrm{~cm}$. The rate of reactions $2^{\prime}$ and 3 ' largely exceeds that of reaction 4 so that instabilities larger than $10^{-5} \mathrm{~cm}$ cannot be eliminated by surface diffusion of $\mathrm{Ag}$ adatoms resulting in dendritic patterns.

The competitive growth of dendrites results in only a few large trees with a size greater than $\langle\delta\rangle$. At this stage, deposit features can be modeled by both the concentration (small-scale features) and electric (large-scale features) fields, as described in 3.2.2. Finally, because of the contribution of a nonlinear term in the interface motion (eq 2), which enhances lateral growth, a transition from a DLA-like to ballistic morphology can be observed.

4.2. Ag Electrodeposition in the Presence of Thiourea. 4.2.1. Validity of the Edwards-Wilkinson Equation. At $j=0.01 \mathrm{~mA} \mathrm{~cm}^{-2}(\approx 0.03 \mathrm{ML} / \mathrm{s}) \mathrm{Ag}$ electrodeposition in a TU-containing solution is accompanied by the appearance of random instabilities (protrusions) at certain surface sites that decay with time. It suggests that preferential adsorption of TU at these instabilities hinder their growth as compared to valleys and flat deposit regions..$^{9,25}$ There are two main pieces of evidence to support this view. The first comes out from autoradiography and counting techniques, ${ }^{26}$ revealing that TU is preferentially adsorbed at protrusions. The second is that TU adsorption is a relatively slow process. In fact, control experiments were made for evaluating the degree of the TU-free surface area of Ag electrodeposits $(1-\theta)$ first grown at $j=0.01 \mathrm{~mA} \mathrm{~cm}{ }^{-2}$ for $t=30$ min using $\mathrm{Pb}$ underpotential deposition (upd). ${ }^{27}$ The adsorption of TU was made from a $0.1 \mathrm{mM}$ TU aqueous solution for different adsorption times $\left(t_{\mathrm{a}}\right)$. Then, the value of $(1-\theta)$ was obtained from the ratio $q\left(t_{\mathrm{a}}\right) / q\left(t_{\mathrm{a}}=0\right), q$ being the voltammetric charge related to $\mathrm{Pb}$ upd (Figure 6a). The degree of the TU-covered Ag electrodeposit surface area $\theta$ vs $t_{\mathrm{a}}$ plot (Figure 6b) shows that for $t_{\mathrm{a}}<600 \mathrm{~s}$ only $20-25 \%$ of the Ag electrodeposit surface area is covered by TU. For $t>600 \mathrm{~s}, \theta$ increases slowly with $t_{\mathrm{a}}$, approaching 0.50 for $t_{\mathrm{a}}=1800 \mathrm{~s}$. This value of $\theta$ is very close to that already reported for the adsorption of TU on $\mathrm{Au} .{ }^{28}$ It should be noted that, even for $\mathrm{Pt}$, a metal surface that is more efficient than the Ag and Au surface for the adsorption of organic molecules, a complete surface coverage by TU adsorbates from a $0.1 \mathrm{mM}$ TU aqueous solution at room tempetature is reached only after $10 \mathrm{~s} .{ }^{29}$ Therefore, for the Ag electrodeposition rate $0.03 \mathrm{ML} \mathrm{s}^{-1}$ that implies $t_{\mathrm{a}}$ $=30 \mathrm{~s}$, it results in $\theta \cong 0.2$. Therefore, from these results and data reported in ref 26 it is reasonable to admit that adsorbed TU molecules are preferentially located at protrusions. The physical reason for the TU adsorption enhancement at protrusions is not clear.

(25) Oniciu, L.; Muresan, L. J. Appl. Electrochem. 1991, 21, 565, and references therein.

(26) Rogers, G. T.; Ware, M. J.; Fellows, R. V. J. Electrochem. Soc. 1960, 107,677 .

(27) Hernandez-Creus, A.; Carro, P.; Gonzalez, S.; Salvarezza, R. C.; Arvia, A. J. J. Electrochem..Soc. 1992, 139, 1064.

(28) Hoelze, M. H.; Apsel, C. W.; Will, T.; Kolb, D. M. J. Electrochem Soc. 1995, 142, 3741.

(29) Bolzan, A. private communication.
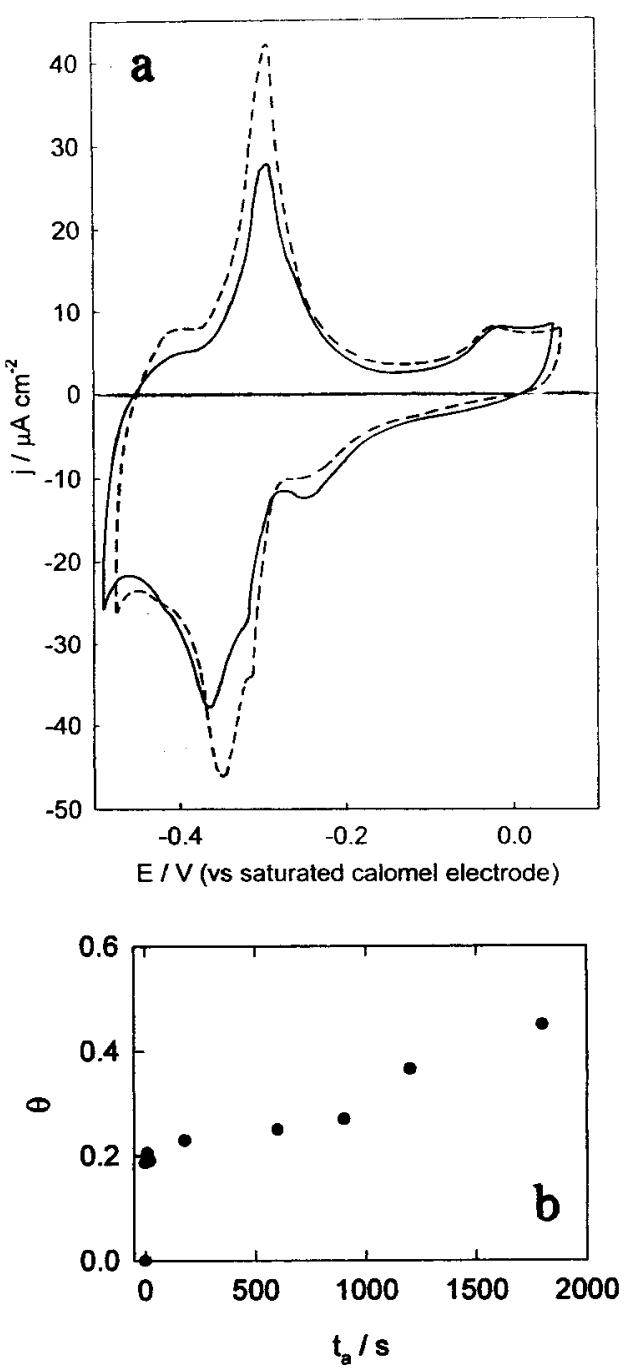

Figure 6. (a) Voltammogram of $\mathrm{Pb}$ underpotential on a $\mathrm{Ag}$ electrodeposit grown at $j=0.01 \mathrm{~mA} \mathrm{~cm}^{-2}$ for $t=30 \mathrm{~min}$. Scan rate $=0.01 \mathrm{~V} \mathrm{~s}^{-1}$. Electrolyte: $10^{-2} \mathrm{M} \mathrm{Pb}(\text { acetate) })_{2}+0.5 \mathrm{M}$ $\mathrm{NaClO}_{4}+10^{-2} \mathrm{MHClO}_{4}$. (a) $t_{\mathrm{a}}=0 \mathrm{~s}$ (dashed trace, TU adsorbatefree voltammogram). (b) $t_{\mathrm{a}}=20 \mathrm{~s}$ (full trace). Before the $\mathrm{Pb}$ underpotential runs TU was adsorbed from a $0.1 \mathrm{mM}$ TU solution for $t_{\mathrm{a}}$. (b) $\theta$ vs $t_{\mathrm{a}}$ plot derived from $\mathrm{Pb}$ upd voltammetry.

Presumably, this is related to the fact that the small radius of curvature at protrusions favors a greater $\mathrm{TU}-\mathrm{Ag}$ interaction. If this is the case, then as the protrusion decays and the radius of curvature increases, TU desorption gradually occurs. According to this model the overall process can be represented by reactions $2^{\prime}-4$ coupled with local TU adsorption/desorption processes:

$$
\mathrm{Tu}(\text { sol }) \leftrightarrow \mathrm{TU}(\text { ads }) \operatorname{Ag}(- \text { surf })
$$

Reaction 5 represents the curvature-dependent adsorption/desorption of TU on Ag.

It should be noted that the smoothening effect observed in the presence of TU cannot be assigned to an enhanced surface diffusion of $\mathrm{Ag}$ adatoms (reaction 4) caused by the presence of $\mathrm{TU}$, as it is known that adsorbed organic molecules tend to decrease the diffusion length of metal adatoms. ${ }^{30}$ Then, the origin of the smoothening effect should be related to the competition of the $\mathrm{TU}$ and $\mathrm{Ag}^{+}$ ion for attachment to protrusion sites. The blocking of protrusions by TU explains the enhanced flux of deposited

(30) Chailapakul, O.; Sun, L.; Xu, C.; Crooks, R. M. J. Am. Chem. Soc. 1993, 115, 12459. 
material to valleys and, consequently, the validity of the EW equation.

The fact that the EW equation turns out to be valid to describe Ag electrodeposition under mass-transport control as well as $\mathrm{Cu}$ electrodeposition under charge-transfer control at low $j$ (region $\mathrm{I}$ ) and in the presence of $\mathrm{TU}^{9}$ indicates that the smoothening mechanism appears to be independent of both the nature of the metal and the kinetic control involved in the process. However, despite these differences there is also a common feature for both as they develop unstable growth fronts in TU-free solutions. For $\mathrm{Cu}$, the microscopic origin of the unstable growth has been related to the enhanced electrodeposition at protrusions where a lower cathodic overpotential is involved..$^{9,31}$ Otherwise, for Ag, the microscopic origin of the unstable growth front should be related mainly to the enhanced capture of $\mathrm{Ag}^{+}$ions by protrusions. ${ }^{3}$ On the other hand, the resulting preferred adsorption of TU at merging protrusions decreases the growth rate there as compared to that of flat regions and valleys by hindering the electron transfer through the adsorbed layer. ${ }^{32}$ This results in a leveling effect of the growth front leading to $W \propto L^{\alpha}$ and $W \propto t^{\beta}$ with $\alpha \approx \beta \approx 0$, as predicted by the $\mathrm{EW}$ equation in $3 \mathrm{D} .{ }^{2}$ These results can be understood considering that in the presence of TU the value of $w$ becomes much smaller than $d_{\mathrm{ac}}$ so that a 3D system is approached.

4.2.2. Development of an Unstable Growth Front at $\boldsymbol{j}=\mathbf{1} \mathbf{m A} / \mathbf{c m}^{-2}$. The situation appears to be more complicated when results from $j=1 \mathrm{~mA} \mathrm{~cm}^{-2}\left(3 \mathrm{ML} \mathrm{s}^{-1}\right)$ are considered. In this case, profiles of Ag electrodeposits exhibit some features that are qualitatively comparable to those obtained in the TU-free solution, even for the highest value of $\mathbf{x}$. In both cases, the two stages of growth can be distinguished, although the density of instabilities, and correspondingly, the density of trees become appreciably diminished for a TU-containing solution (Figure 5). Likewise, the ratio of the number of random instabilities to the number of growing trees increases as $\mathbf{x}$ is increased. The reverse effect is noticed for the number of sites with branches initially grown that cease to grow (dead branches).

The Ag electrodeposit profiles at high $j$ (Figure 5) can also be explained in terms of competitive reactions [ $\left(2^{\prime}\right.$ and $\left.\left.3^{\prime}\right)\right]$ and (5). At the early stages of growth the rate of Ag electrodeposition is much faster than the adsorption rate of TU at protrusions. For $3 \mathrm{ML} \mathrm{s}^{-1} t_{\mathrm{a}}=0.3 \mathrm{~s}$, TU surface coverage is small (Figure 6b), and no full coverage at protrusions is attained. This means that the electrodeposition at protrusions continues over tiny TU-free surfaces sustaining branching as local diffusion fields due to both $\mathrm{Ag}^{+}$ions and TU are built up at each growing protrusion. Accordingly, the driven force for the desorption/ adsorption displacement of TU (reaction 5) tends to be suppressed. As neighbor trees further grow, the overlap-

(31) Despic, A. R.; Popov, K. I. In Modern Aspects of Electrochemistry; Bockris, J.O.', Conway, B. E., Eds.; Butterworths: New York, 1972; Vol. 7, p 199

(32) Sabatini, E.; Rubinstein, J. J. Phys. Chem. 1987, 91, 6663. ping of their diffusion layers caused by depletion of $\mathrm{Ag}^{+}$ ions at the interface takes place. At this stage, the development of large trees produces a shadowing effect for the growth of small trees. Consequently, the TU concentration gradient at the top of small trees tends to disappear, protrusion surfaces become covered by TU, and finally, the growth rate of small trees turns out to be extremely low. Moreover, the development of large trees implies a large fractal surface, ${ }^{1,2}$ which means a large adsorption area, and concomitantly, a TU concentration gradient sets in around the outer parts of large trees together with the concentration gradient of depositing $\mathrm{Ag}^{+}$ions.

The overall effect is that, at advanced stages of growth, Laplacian fields (concentration and electrical) assist the development of branched morphologies that can be described by fractal geometry. In this case, the interface evolution follows the same relationship as that found in the absence of the additive. The interpretation given above explains why the presence of TU fails to suppress completely the development of instabilities even at the highest concentration.

\section{Conclusions}

The adsorption of TU on a Ag electrodeposit growing at a low current density $\left(j=0.01 \mathrm{~mA} \mathrm{~cm}^{-2}\right)$ from acid solution containing a supporting electrolyte eliminates instabilities. The smoothing of the electrodeposit can be explained by the preferred adsorption of TU at protrusions, impeding further growth there. Smoothing can be explained by TU adsorption/desorption processes occurring locally following the random appearance of instabilities. In this case, the interface dynamics can be well-described by the EW equation. The model recently proposed to explain the smoothening effect of TU on the activation-controlled electrodeposition of $\mathrm{Cu}$ can be extended to the masstransport-controlled electrodeposition of Ag.

The electrodeposition of $\mathrm{Ag}$ in the presence of TU at a high current density $\left(j=1 \mathrm{~mA} \mathrm{~cm}^{-2}\right)$ shows branching growth as observed in a TU-free solution. The presence of TU decreases the rate of nucleation and growth of instabilities, but fails to suppress completely their formation. The overall result leads to the development of an unstable growth front. In this case, the fast Ag electrodeposition reaction impedes the adsorption equilibrium for $\mathrm{TU}$, and consequently, the driven force for the adsorption/desorption displacement of TU tends to be suppressed. Branching is assisted by the local electric field and concentration fields resulting from the depletion of $\mathrm{Ag}^{+}$ions and TU molecules at the growth front.

Acknowledgment. This work was financially supported by PIA 7283/97 and PIP 014/97 from the Consejo Nacional de Investigaciones Científicas y Técnicas (CONICET) of Argentina.

LA9806092 